\title{
Otimização de parâmetros estruturais, dielétricos e ferroelétricos de filmes finos de PZT
}

\section{(Optimization of structural, dielectric and ferroelectric parameters of PZT thin films)}

\author{
E. B. Araújo, J. A. Eiras \\ Departamento de Física - Grupo de Cerâmicas Ferroelétricas \\ Universidade Federal de S. Carlos - UFSCar \\ C. P. 676, S. Carlos, SP, 13565-670
}

\section{Resumo}

Este trabalho faz uma síntese de resultados obtidos para filmes finos de PZT processados por diferentes vias com objetivo de otimizar parâmetros estruturais, dielétricos e ferroelétricos. Filmes foram preparados em fornos convencionais e pelo método de tratamento térmico rápido (TTR). Os resultados foram comparados e mostraram que os filmes cristalizados usando TTR apresentaram uma melhor cristalização, comparado com os filmes cristalizados em forno convencional. Em consequiência, melhores parâmetros dielétricos e ferroelétricos também foram obtidos, chegando a duplicar o valor da polarização remanescente $\left(\mathrm{P}_{\mathrm{r}}\right)$ e aumentar significativamente a constante dielétrica $(\varepsilon)$ dos filmes.

Palavras-chave: filmes finos, PZT, ferroelétrico.

\section{INTRODUÇÃO}

O Titanato Zirconato de Chumbo, $\mathrm{PbZr}_{\mathrm{x}} \mathrm{Ti}_{1-\mathrm{x}} \mathrm{O}_{3}$ (PZT), provavelmente tem sido um dos materiais ferroelétricos mais estudados nos últimos anos, tanto na forma de corpos cerâmicos como também filmes finos. Corpos cerâmicos de PZT tornaram-se conhecidos pelas excelentes características dielétricas, piezoelétricas e piroelétricas [1 ]. Nos últimos tempos, com o crescente avanço da tecnologia da microeletrônica, o PZT despontou como um material atrativo que foi amplamente utilizado na forma de filmes finos, particularmente em dispositivos de memória para computadores.

Com a crescente evolução da tecnologia de microcircuitos diversificam-se os métodos para a obtenção de filmes finos. No caso específico do PZT, filmes finos têm sido depositados por $D C$ sputtering [2], RF sputtering [3, 4], Sol Gel [5] entre outros. Entre os várias métodos para a preparação de filmes finos ferroelétricos os processos químicos destacam-se como rotas promissoras pois permitem melhor controle estequiométrico de compostos complexos do que métodos físicos como RF sputtering ou laser ablation [6].

Recentemente, propusemos uma rota alternativa para a preparação de filmes finos ferroelétricos partindo do óxido calcinado [7]. Trata-se de um método químico que permitiu a obtenção de filmes finos de boa qualidade e excelente controle estequiométrico. Os primeiros resultados obtidos para filmes de PZT [8], embora concordem com aqueles divulgados pela literatura, estimularam a conseqüente otimização de alguns parâmetros, como

Abstract
This work make synthesis of results obtained from PZT thin
films processed by different ways to optimizes structural,
dielectric and ferroelectric parameters. Films were prepared
using conventional furnace and rapid thermal annealing
$(R T A)$. Results were compared and showing that films
crystallized by RTA showed better crystallization, if compared
with films crystallized by conventional furnace. Thus, better
dielectric and ferroelectric parameters were also obtained,
duplicating values of remanent polarization $\left(P_{r}\right)$ and
increasing substantially the dielectric constant $(\varepsilon)$ of the films.
Keywords: thin films, PZT, ferroelectric.

os dielétricos e ferroelétricos, que viabilizariam a futura aplicação em dispositivos.

O controle da cristalização é um fator importante na tecnologia de filmes finos. Considerando filmes finos ferroelétricos na microeletrônica, um dos principais requisitos resume-se a obter um material com boas características estruturais, dielétricas e ferroelétricas. Essas características podem ser melhoradas controlando-se adequadamente o processo de cristalização, o tamanho de grãos, dentre outros.

Esse trabalho teve como objetivo otimizar parâmetros estruturais, dielétricos e ferroelétricos de filmes finos de PZT preparados pelo método alternativo descrito anteriormente [7]. A otimização destes parâmetros foi avaliada sob aspectos da preparação dos filmes, investigando-se filmes cristalizados em forno convencional e também filmes cristalizados pelo técnica de tratamento térmico rápido (TTR). Essa última técnica apresenta algumas vantagens sobre o tratamento convencional, dentre elas a redução do tempo de processamento de horas para apenas alguns segundos. A estrutura cristalina dos filmes foi investigada por meio de difração de raios X, os parâmetros dielétricos por meio de espectroscopia de impedância e os parâmetros ferroelétricos por meio de ciclos de histerese.

\section{PROCEDIMENTO EXPERIMENTAL}

Os filmes de PZT utilizados no estudo deste trabalho foram preparados com a razão molar $\mathrm{Zr} / \mathrm{Ti}=53 / 47$. Nessa composição 
muitas das propriedades do PZT como constante dielétrica, coeficientes piezoelétricos e eletromecânicos, apresentam seus máximos valores [1]. Usando o método descrito em nosso recente artigo [7], filmes de resina polimérica foram depositados, a temperatura ambiente, sobre substratos de Si e Pt/Si spin coating. Os substratos foram limpos previamente em uma solução de água e detergente sob aquecimento por 20 minutos a $70{ }^{\circ} \mathrm{C}$, seguido de uma limpeza em ultra-som por 10 minutos. Finalmente os substratos foram enxaguados em água destilada e secos em chapa quente.

Com os substratos limpos resinas poliméricas foram depositadas sobre $\mathrm{Si}$ e Pt/Si a uma rotação de $5000 \mathrm{rpm}$ por 40 a 50 segundos. Os filmes de resina foram aquecidos em forno convencional a 350 ${ }^{\circ} \mathrm{C}$ durante 5 horas para a remoção de material orgânico. O processo foi repetido até que seis camadas foram depositadas para atingir a espessura desejada antes do tratamento térmico para a cristalização. Os filmes foram cristalizados em forno convencional a $700{ }^{\circ} \mathrm{C}$ por 1 hora e pela técnica TTR a $700{ }^{\circ} \mathrm{C}$ por 60 segundos. Os filmes cristalizados apresentaram boa aderência e uniformidade em ambos os substratos. As espessuras dos filmes foram determinadas utilizando-se um perfilômetro Mytutoyo 178-896D, medindo-se a altura do degrau formado entre cada filme e o substrato. As espessuras dos filmes determinadas estiveram em torno de 520 e $550 \mathrm{~nm}$. A estrutura cristalográfica dos filmes foram estudadas por análises de difração de raios $\mathrm{X}$, usando radiação $\mathrm{CuK} \alpha$ à temperatura ambiente.

As propriedades dielétricas incluem medidas da constante dielétrica e fator de dissipação em função da freqüência. Esses ensaios foram realizados usando um analisador de impedâncias HP 4194A entre as frequiências de $100 \mathrm{~Hz}$ e $10 \mathrm{MHz}$. As caracterizações ferroelétricas incluíram medidas de ciclos de histereses P-E para a frequiência de $100 \mathrm{~Hz}$, usando um circuito Sawyer-Tower acoplado a osciloscópio digital Tektronix 2232. Para medir os parâmetros dielétricos e ferroelétricos filmes de PZT foram cristalizados sobre substratos de Pt/Si e vários eletrodos de ouro (0,3 mm de diâmetro) foram depositados por DC Sputtering, em uma área de $1 \mathrm{~cm}^{2}$ sobre o PZT, através de uma máscara, formando a configuração Au/PZT/ $\mathrm{Pt} / \mathrm{Si}$. Dessa forma, uma matriz de $10 \times 10$ capacitores foi obtida, constituindo o ouro o eletrodo superior e a platina o eletrodo inferior.

\section{RESULTADOS E DISCUSSÃO}

A Fig. 1-A apresenta um difratograma de raios $\mathrm{X}$ de um filme de PZT depositado sobre Si e tratado termicamente a $700{ }^{\circ} \mathrm{C}$ por 2 horas, usando um forno convencional. Nesta figura foi identificada a coexistência das fases tetragonal e romboédrica, conforme indicam os picos indexados. Os parâmetros de rede foram calculados a partir do difratograma da Fig. 1-A para ambas as fases usando-se o método dos mínimos quadrados. Os $a_{T}$ e $c_{T}$ da fase tetragonal do PZT foram calculados considerando-se os picos $(100)_{\mathrm{T}},(101)_{\mathrm{T}},(110)_{\mathrm{T}} \mathrm{e}(210)_{\mathrm{T}}$. Os valores $a_{T}$ e $c_{T}$ foram respectivamente 4,02 e 4,18 $\AA$. A razão $c_{\mathrm{T}} /$ $a_{\mathrm{T}}$ fornece o grau de tetragonalidade. Com base nos resultados obtidos obtêm-se $c_{\mathrm{T}} / a_{\mathrm{T}}=1,04$. Essa razão concorda bem com resultados obtidos para corpos cerâmicos de PZT com a mesma composição [1] como também filmes finos produzidos, por exemplo, por DC sputtering [2]. Para a fase romboédrica do PZT foram obtidos os parâmetros $a_{R}=4.08 \AA$ e $90-\alpha=0.024^{\circ}$, considerandose os picos $(101)_{\mathrm{R}},(111)_{\mathrm{R}}$ and $(200)_{\mathrm{R}}$.

A Fig. 1-B apresenta difratograma de raios X para filme de PZT

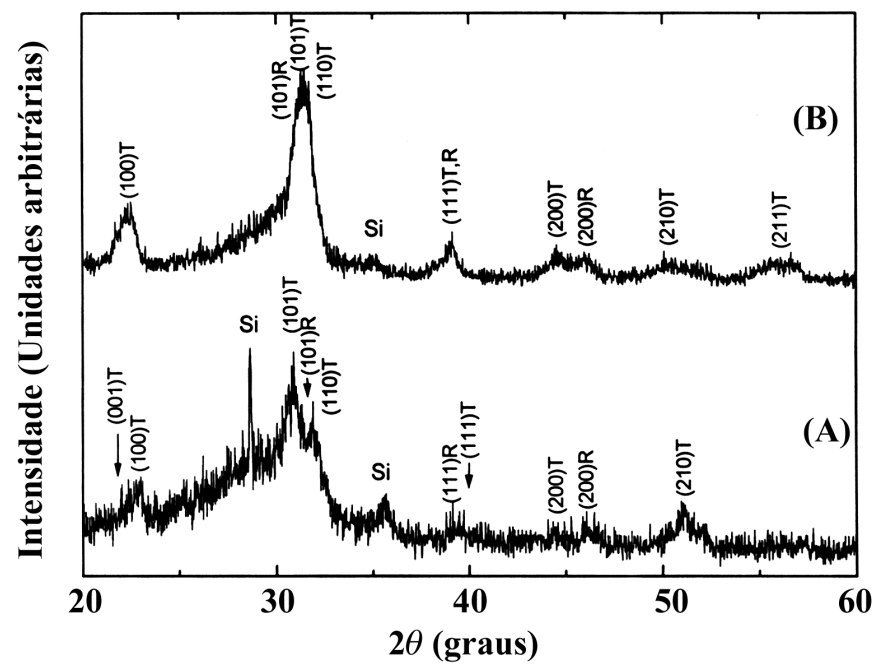

Figura 1: Difratogramas de raios $\mathrm{X}$ para filmes depositados sobre $\mathrm{Si}$, cristalizados a $700{ }^{\circ} \mathrm{C}$ por 2 horas (A) e $700{ }^{\circ} \mathrm{C}$ por 60 segundos (B), respectivamente pelos métodos convencional e TTR.

[Figure 1: X-Ray pattern of films deposited on Si, crystallized at $700^{\circ} \mathrm{C}$ for 2 hours (A) and $700^{\circ} \mathrm{C}$ for 60 seconds (B), by conventional method and RTA, respectively].

depositado sobre $\mathrm{Si}$ e cristalizado a $700^{\circ} \mathrm{C}$ por 60 segundos, usando TTR. Neste filme verificou-se a cristalização das fases tetragonal e romboédrica, tal qual observado no filme tratado em forno convencional. Os parâmetros de rede para este filme, calculados com base nos mesmos picos, para a fase tetragonal foram $a_{T}=4.04$ e $c_{T}=$ $4.06 \AA$ e para a fase romboédrica foram $a_{R}=4.05 \AA$ e $90-\alpha=0.016^{\circ}$. $\mathrm{O}$ grau de tetragonalidade medido foi $c_{\mathrm{T}} / a_{\mathrm{T}}=1.005$.

Com base nos resultados anteriores vê-se que o grau de tetragonalidade do filme cristalizado em forno convencional é maior do que o filme cristalizado por TTR e que o volume da célula unitária da fase tetragonal $\left(67,55 \AA^{3}\right)$, para o filme cristalizado em forno convencional, é ligeiramente maior do que para o filme cristalizado usando TTR $\left(66,27 \AA^{3}\right)$. Com respeito a fase romboédrica a distorção observada foi menor nos filmes preparados por TTR. Portanto, filmes cristalizados por TTR apresentaram uma célula tetragonal mais compacta e uma menor distorção da fase romboédrica. A aparente efetiva cristalização do PZT usando a técnica TTR pode ser atribuída ao rápido equilíbrio atingido durante o processo de cristalização. Neste caso, a temperatura desejada para a cristalização é atingida em um tempo muito pequeno, sendo também pequeno o tempo no qual o filme fica exposto em alta temperatura. De certa forma isto acarreta em uma redução da interação filme-substrato e também minimiza os efeitos de superfície. Nesse trabalho, a técnica TTR teve uma influência direta e permitiu a cristalização do PZT sem a presença da fase pirocloro, o que foi também observado em filmes de PZT processados por outros métodos químicos [9].

Com base nas Figs. 2-A e 2-B (filmes depositados sobre $\mathrm{Pt} / \mathrm{Si}$ ), também foram calculados os parâmetros das fases tetragonal e romboédrica. Para o filme cristalizado pelo método convencional (Fig. 2-A) os parâmetros da fase tetragonal foram $a_{\mathrm{T}}=4,05 \AA$ e $c_{\mathrm{T}}$ $=4,08 \AA\left(a_{\mathrm{T}} / c_{\mathrm{T}} \cong 1,01\right)$, e para o filme cristalizado por TTR (Fig. 2B), os parâmetros encontrados foram $a_{\mathrm{T}}=4,07 \AA \mathrm{A}$ e $c_{\mathrm{T}}=4,09 \AA\left(a_{\mathrm{T}}\right)$ $\left.c_{\mathrm{T}} \cong 1,01\right)$. Os picos da fase romboédrica não estão bem definidos na Fig. 2. Portanto, para os filmes depositados sobre Pt/Si somente foram calculados os parâmetros da fase tetragonal do PZT. Esses resultados permitem constatar que o grau de tetragonalidade é 


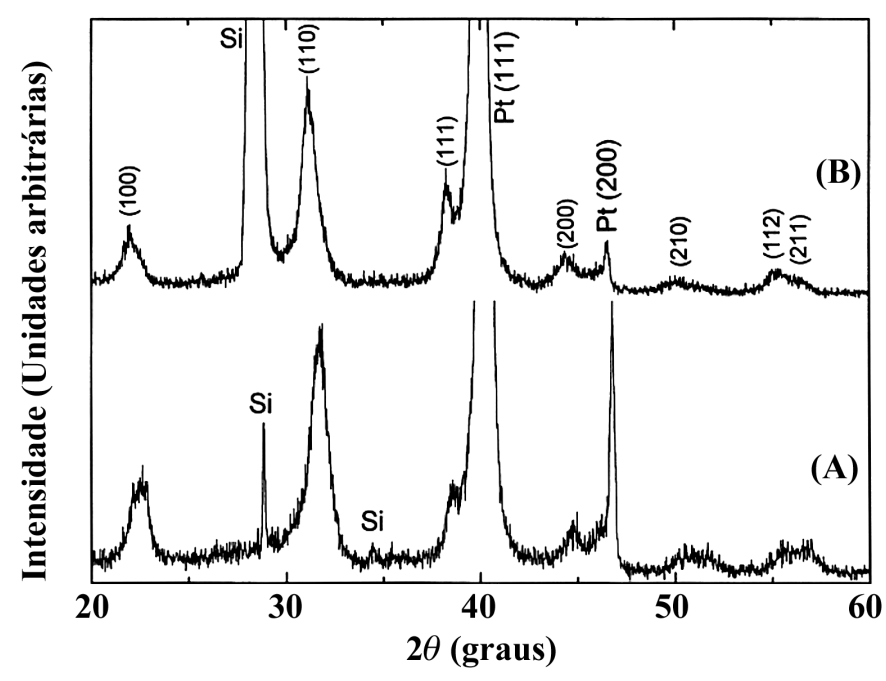

Figura 2: Difratogramas de raios $\mathrm{X}$ para filmes depositados sobre sobre $\mathrm{Pt} / \mathrm{Si}$, cristalizados a $700{ }^{\circ} \mathrm{C}$ por 1 hora (A) e $700^{\circ} \mathrm{C}$ por 60 segundos (B), pelos métodos convencional e TTR.

[Figure 2: X-Ray pattern of films deposited on Pt/Si, crystallized at $700^{\circ} \mathrm{C}$ for 1 hour (A) and $700^{\circ} \mathrm{C}$ for 60 seconds (B), by conventional and RTA method].

essencialmente o mesmo para os filmes cristalizados pelas diferentes vias e depositados sobre $\mathrm{Pt} / \mathrm{Si}$. Para os filmes depositados sobre $\mathrm{Si}$, com base nos resultados, o efeito do processamento rápido aparentemente provoca uma maior distorção na célula unitária, ao contrário do que foi observado para os filmes depositados sobre $\mathrm{Pt} / \mathrm{Si}$. Os valores obtidos para os parâmetros de rede, entretanto, estão em boa concordância com resultados da literatura para filmes de PZT processados por outros métodos químicos como decomposição de orgânicos metálicos [9].

A Fig. 3 ilustra o comportamento da constante dielétrica e do fator de dissipação em função da freqüência $(100 \mathrm{~Hz}$ a $10 \mathrm{MHz})$, para filme de PZT cristalizado pelo método convencional a $700{ }^{\circ} \mathrm{C}$ por 1 hora (Fig. 3-A) e por TTR a $700{ }^{\circ} \mathrm{C}$ por 60 segundos (Fig. 3-
B). Dois aspectos importantes podem ser observados, como efeito do processamento dos filmes de PZT. O primeiro está associado à dispersão observada para freqüências superiores a $100 \mathrm{kHz}$. Para o filme preparado pelo método convencional a dispersão é pronunciada, no intervalo de $100 \mathrm{kHz}$ a $10 \mathrm{MHz}$ o valor da constante dielétrica cai de 358 para 144 e o fator de dissipação aumenta consideravelmente de 0,039 até 0,743 . Por outro lado, no filme preparado por TTR esse efeito não foi observado, o valor da constante dielétrica caiu linearmente no intervalo medido (737 a 576). O outro aspecto está associado com o valor da constante dielétrica $(\varepsilon)$. No intervalo de freqüências medido os valores de $\varepsilon$ foram sempre maiores para os filmes preparados por TTR do que para os filmes obtidos pelo método convencional.

A dispersão observada a altas freqüências tem sido freqüientemente observada em outros filmes ferroelétricos [10]. O fato de não ter sido observado aqui para os filmes preparados por TTR não implica necessariamente que a dispersão não exista. Provavelmente ela foi deslocada para freqüências superiores, o que supostamente decorre da redução de efeitos de interface, melhor controlado nos filmes preparados por TTR. Por outro lado, os valores obtidos para a constante dielétrica, em particular para o filme preparado por TTR, concordam bem com os valores divulgados na literatura para filmes de PZT produzidos por outros métodos químicos consolidados.

Os parâmetros ferroelétricos foram obtidos a partir de ciclos de histereses dos filmes depositados sobre substratos de $\mathrm{Pt} / \mathrm{Si}$ e em forno convencional e pela técnica de TTR. As Figs. 4-A e 4-B apresentam ciclos de histereses para filmes cristalizados a $700{ }^{\circ} \mathrm{C}$ por 1 hora e a $700{ }^{\circ} \mathrm{C}$ por 60 segundos, respectivamente em forno convencional e por TTR. A partir da Fig. 4-A foram determinadas a polarização remanescente $\left(P_{r}\right)$ e o campo coercitivo $\left(E_{c}\right)$, respectivamente iguais a $7,8 \mu \mathrm{C} / \mathrm{cm}^{2}$ e $99 \mathrm{kV} / \mathrm{cm}$. Por outro lado, considerando-se a Fig. 4-B os valores obtidos foram $P_{r}=15,7 \mu \mathrm{C}$ / $\mathrm{cm}^{2}$ e $E_{c}=73 \mathrm{kV} / \mathrm{cm}$. Esses valores concordam com aqueles obtidos na literatura e para filmes de PZT com a mesma composição os

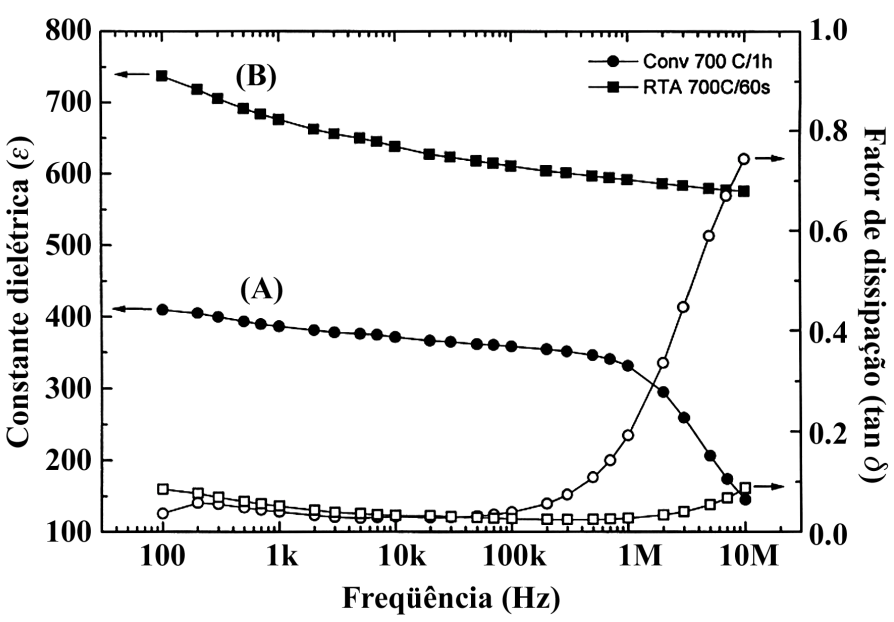

Figura 3: Constante dielétrica e fator de dissipação em função da freqüência para filmes de PZT cristalizados a $700{ }^{\circ} \mathrm{C}$ por 1 hora (A) e a $700{ }^{\circ} \mathrm{C}$ por 60 segundos (B), respectivamente pelos métodos convencional e TTR. Os filmes foram depositados sobre $\mathrm{Pt} / \mathrm{Si}$.

[Figure 3: Dielectric constant and dissipation factor as a function of frequency for PZT films crystallized at $700^{\circ} \mathrm{C}$ for 1 hour (A) and at $700^{\circ} \mathrm{C}$ for 60 seconds $(B)$, respectively by conventional and RTA method. Films were deposited on Pt/Si].

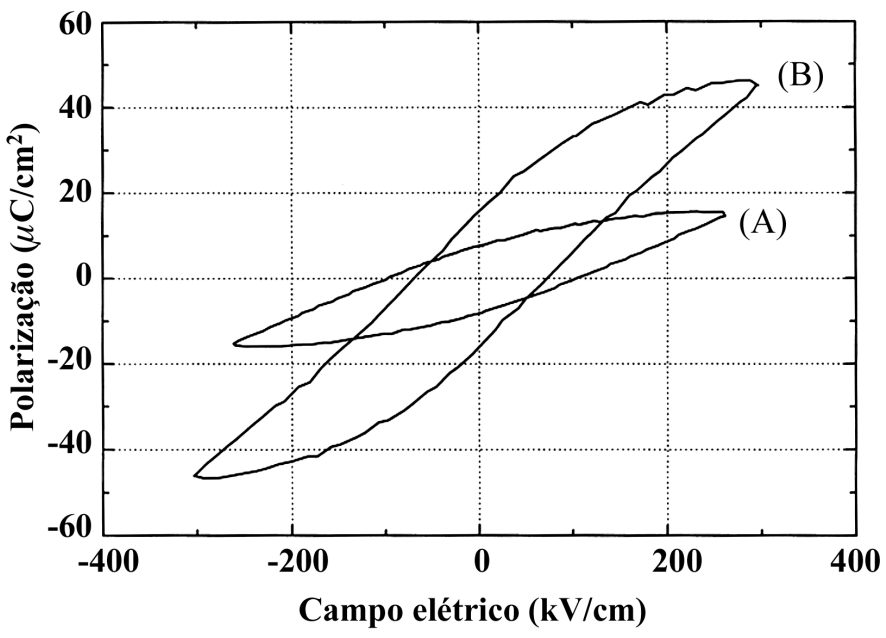

Figura 4: Ciclos de histerese obtidas a $100 \mathrm{HZ}$ para filmes de PZT cristalizados a $700{ }^{\circ} \mathrm{C}$ por 1 hora (A) e a $700{ }^{\circ} \mathrm{C}$ por 60 segundos (B), pelos métodos convencional e TTR (filmes depositados sobre Pt/Si).

[Figure 4: Hysteresis loops obtained at $100 \mathrm{HZ}$ for PZT films crystallized at $700^{\circ} \mathrm{C}$ for 1 hour (A) and $700^{\circ} \mathrm{C}$ for 60 seconds (B), by conventional and RTA method (films were deposited on $\mathrm{Pt} / \mathrm{Si}$ )]. 
valores de $P_{r}$ e $E_{c}$ estão respectivamente no intervalo $1-7 \mu \mathrm{C} / \mathrm{cm}^{2} \mathrm{e}$ $26-80 \mathrm{kV} / \mathrm{cm}$, obtidos por Sol Gel [5] ou 3-30 $\mu \mathrm{C} / \mathrm{cm}^{2}$ e $25-64 \mathrm{kV} /$ $\mathrm{cm}$, para filmes obtidos por DC Sputtering [2].

Como observado, o valor de $P_{r}$ apresentado pelo filme cristalizado por TTR é pelo menos duas vezes maior do que o valor obtido para o filme cristalizado em forno convencional, enquanto o campo coercitivo foi reduzido a $2 / 3 \mathrm{em}$ magnitude.

\section{CONCLUSÕES}

Filmes finos de PZT, preparados em fornos convencionais e pelo método TTR, apresentaram distintas características estruturais, dielétricas e ferroelétricas. Os parâmetros dielétricos e ferroelétricos sofreram grande influência em decorrência do processamento. O comportamento da constante dielétrica para os filmes preparados pelo método TTR foi melhor controlado do que para os filmes preparados pelo método convencional, que apresentou uma indesejável dispersão a altas freqüências. Com respeito às características ferroelétricas, os filmes preparados por TTR apresentaram maior polarização remanescente e menor campo coercitivo, se comparado aos filmes preparados em forno convencional. Esses resultados sugerem a viabilidade na preparação de filmes finos pelo método de tratamento térmico rápido (TTR), levando a desejáveis parâmetros dielétricos e ferroelétricos, em decorrência da otimização do processamento desses filmes.

\section{AGRADECIMENTOS}

Agradecemos à Dra. Yvonne P. Mascarenhas (IFSC-USP), pelas facilidades com o difratômetro de raios X e ao CNPq e a FAPESP (processo 99/02485-2) pelo suporte financeiro.

\section{REFERÊNCIAS}

[1] B. Jaffe, W. R. Cook, H. Jaffe, Piezoelectric Ceramics, Academic, New York (1971).

[2] K. Sreenivas, M. Sayer, J. Appl. Phys. 64, 3 (1988) 1484.

[3] K. Sreenivas, M. Sayer, P. Garret, Thin Solid Films, 172, 2 (1989) 251.

[4] R. Takayama, Y. Tomita, J. Appl. Phys. 65, 4 (1989) 1666.

[5] G. Yi, Z. Wu , M. Sayer, J. Appl. Phys. 64, 5 (1988) 2717.

[6] O. Auciello, L. Mantese, J. Duarte, X. Chen, S. H. Rou, A. I. Kingon, A. F. Schreiner, A. R. Krauss, J. Appl. Phys. 73, 10 (1993) 5197.

[7] E. B. Araújo, J. A. Eiras, J. Mat. Sci. Letters 17, 10 (1998) 833. [8] E. B. Araújo, J. A. Eiras, J. Phys.: Cond. Matter 11, 8 (1999) 1975. [9] H. Hu, L. Shi, V. Kumar, S. B. Krupanidhi, Ceramic Transactions, Ferroelectric films, Edited by A. S. Bhalla, K. M. Nair, 25 (1992) 113. [10] P. C. Joshi, S. B. Krupanidhi, J. Appl. Phys. 72 (1992) 5827.

(Rec. 31/07/00, Ac. 02/02/01) 